\title{
Riparian-Wetland \\ Initiative for the 1990's \\ Report of Accomplishments for Fiscal Year (FY) 1998
}

\section{Introduction}

This is the eighth annual accomplishments report of the Riparian-Wetland Initiative for the 1990 's, a blueprint for managing and restoring riparian-wetland areas that cover about 12,932,434 acres of wetlands and 177,052 miles of streams on BLM-managed lands. Overall, riparianwetland areas account for more than 8 percent of the 264 million acres of land under BLM management. This report shows the status of BLM riparian-wetland areas at the end of FY1998.

\section{Importance of Riparian-Wetland Areas}

Riparian areas are lands adjacent to creeks, streams, lakes, and rivers. They are sometimes called "green ribbons" because the vegetation on the banks of waterways forms a ribbon-like pattern when seen from the air. Wetlands are generally defined as areas inundated or saturated by surface or ground water at a frequency and duration sufficient to support vegetation that is typically adapted for life in saturated soil. Wetlands include bogs, marshes, shallows, muskegs, wet meadows, estuaries, and riparian areas. Riparian areas and wetlands are discussed together for purposes of reporting the BLM's progress in meeting its national goals.

Because riparian-wetland areas contain scarce water and vegetation in the otherwise arid Western United States, these areas are important to fish and wildlife species, as well as to livestock.

Riparian-wetland areas act like sponges in the hydrologic cycle; they capture and store high flows and slowly release them during the low flow periods of summer. Since they filter the water flowing through them, riparian-wetland areas contribute to providing clean water for fishing, swimming, municipal, and other uses. Overall, they are important to the health of entire watersheds.

\section{Proper Functioning Condition}

Riparian-wetland areas are healthy and functioning properly when adequate vegetation, land form, or large woody debris is present to dissipate energy associated with high water flows or wave action. Healthy riparian-wetland areas perform several critical functions such as:

-Purifying water by removing sediments;

-Reducing the risk of flood damage;

-Increasing available water by holding it in streambanks and aquifers;

-Maintaining in stream flows;

-Stabilizing stream banks;

-Increasing ground-water supplies;

-Supporting a diversity of wildlife and plant species;

-Maintaining habitats for healthy fish populations;

-Providing water, forage, and shade for livestock; and 
-Creating opportunities for recreationists to fish, swim, camp, picnic, and relax.

Condition of BLM Riparian-Wetland Areas

All the States are moving closer to completing their initial assessments of the condition of riparian-wetland areas on BLM-managed lands. Most high priority management riparian areas have now been assessed. Just over 80 percent of the BLM riparian areas in the lower 48 States have been assessed using the Proper Functioning Condition (PFC) process. About 47percent of BLM's riparian areas in the lower 48 are in Proper Functioning Condition or Functioning-AtRisk with an Upward Trend. An upward trend implies that current management is helping to improve the condition of an area that is in less than proper functioning condition. In Alaska, 91 percent of the BLM riparian areas are in Proper Functioning Condition.

Approximately 44 percent of the wetlands on BLM-managed lands in the lower 48 States remain in the Unknown category; they have not been assessed for functioning condition. However, almost 48 percent of all BLM wetlands in the lower 48 are in Proper Functioning Condition or Functioning-At-Risk with an Upward Trend. That total is 98 percent for Alaska where the vast majority of wetlands are in pristine condition.

Appendix I to this report contains a table which depicts the condition of riparian-wetland areas on BLM-managed lands at the end of FY 1998. The condition classes are shown by State in miles for streams and acres for wetlands. There are four classes: Proper Functioning Condition, Functioning-At-Risk, Non-Functional, and Unknown. These terms are defined in the footnotes to the table.

\section{Workload Summary}

In FY 1998, BLM spent $\$ 16,473,000$ in the Riparian (1040) subactivity. With the help of numerous partners, including sharing some costs with other programs within the BLM, the following workload accomplishments were achieved:

5,109 Riparian Miles Assessed;

20,348 Wetland Acres Assessed; Improvements Made on 1,466 Miles of Riparian Habitat;

465 Riparian-Wetland Projects Built;

785 Riparian-Wetland Projects Maintained;

Monitoring on 863 Miles of Riparian Habitat;

Monitoring on 2,215 Acres of Wetland Habitat;

New Management Initiated on 2,462 Miles of Riparian Habitat;

New Management Initiated on 19,054 Acres of Wetland Habitat.

\section{Conclusion}

The BLM is making significant headway in improving the condition of riparian-wetlands throughout the West. The initiation of Standards and Guidelines on BLM lands and the implementation of several Clean Water Action Plan action items will greatly enhance the protection given to riparian-wetland values in the coming years. 
Responding to the challenges of riparian-wetland restoration and management will require the continued support of Congress and ongoing cooperation between the BLM and its numerous public and private partners. Given this support and cooperation, the BLM will continue to increase the miles and acres of healthy riparian-wetland habitats on public lands. 


\section{APPENDIX I \\ CONDITION OF RIPARIAN-WETLAND AREAS, FY 98}

\begin{tabular}{|c|c|c|c|c|c|c|c|c|c|}
\hline \multirow[t]{2}{*}{ State } & \multirow[t]{2}{*}{$\begin{array}{c}\text { Habitat } \\
\text { Types }\end{array}$} & \multirow{2}{*}{$\begin{array}{l}\text { Proper } \\
\text { Functioning } \\
\text { Condition } \\
\text { /b/ }\end{array}$} & \multicolumn{4}{|c|}{$\begin{array}{l}\text { Functioning-At-Risk } \\
\qquad / \mathrm{c} /\end{array}$} & \multirow{2}{*}{$\begin{array}{l}\text { Non } \\
\text { Functional } \\
\text { /d } /\end{array}$} & \multirow[t]{2}{*}{$\begin{array}{l}\text { Unknown } \\
/ \mathrm{e} /\end{array}$} & \multirow[t]{2}{*}{ Total } \\
\hline & & & $\begin{array}{l}\text { Trend } \\
\text { Up }\end{array}$ & $\begin{array}{c}\text { Trend } \\
\text { Not } \\
\text { Apparent } \\
\end{array}$ & $\begin{array}{l}\text { Trend } \\
\text { Down }\end{array}$ & Total & & & \\
\hline \multirow[t]{2}{*}{$\mathrm{AK}$} & $\begin{array}{l}\text { Riparian } \\
\text { Miles } / \mathbf{f} /\end{array}$ & $\begin{array}{r}127,875 \\
(91 \%)\end{array}$ & 35 & 0 & 0 & $\begin{array}{r}35 \\
\text { (trace) }\end{array}$ & $\begin{array}{r}812 \\
(1 \%)\end{array}$ & $\begin{array}{r}11,639 \\
(8 \%)\end{array}$ & 140,361 \\
\hline & $\begin{array}{l}\text { Wetland } \\
\text { Acres } / g /\end{array}$ & $\begin{array}{r}12,376,200 \\
(98 \%)\end{array}$ & $\mathrm{h} /$ & $/ \mathrm{h} /$ & $/ \mathrm{h} /$ & $/ h /$ & $/ \mathrm{h} /$ & $\begin{array}{r}188,800 \\
(2 \%)\end{array}$ & $12,565,000$ \\
\hline \multirow[t]{2}{*}{$\mathrm{AZ}$} & $\begin{array}{l}\text { Riparian } \\
\text { Miles }\end{array}$ & $\begin{array}{r}290 \\
(34 \%)\end{array}$ & 145 & 221 & 70 & $\begin{array}{r}436 \\
(51 \%)\end{array}$ & $\begin{array}{r}21 \\
(2 \%)\end{array}$ & $\begin{array}{r}113 \\
(13 \%)\end{array}$ & 860 \\
\hline & $\begin{array}{l}\text { Wetland } \\
\text { Acres }\end{array}$ & $\begin{array}{r}98 \\
(\mathrm{~T}) \\
\end{array}$ & 17,830 & 13 & 96 & $\begin{array}{r}17,939 \\
(82 \%) \\
\end{array}$ & 0 & $\begin{array}{r}3,865 \\
(18 \%) \\
\end{array}$ & 21,902 \\
\hline \multirow[t]{2}{*}{$\mathrm{CA}$} & $\begin{array}{l}\text { Riparian } \\
\text { Miles }\end{array}$ & $\begin{array}{r}1,865 \\
(52 \%)\end{array}$ & 395 & 700 & 104 & $\begin{array}{r}1,199 \\
(33 \%)\end{array}$ & $\begin{array}{r}101 \\
(3 \%)\end{array}$ & $\begin{array}{r}425 \\
(12 \%)\end{array}$ & 3,590 \\
\hline & $\begin{array}{l}\text { Wetland } \\
\text { Acres }\end{array}$ & $\begin{array}{r}11,273 \\
(85 \%)\end{array}$ & 3,100 & 6,516 & 955 & $\begin{array}{r}10,571 \\
(12 \%)\end{array}$ & $\begin{array}{r}413 \\
(2 \%)\end{array}$ & $\begin{array}{r}237 \\
(1 \%)\end{array}$ & 22,494 \\
\hline \multirow[t]{2}{*}{$\mathrm{CO}$} & $\begin{array}{l}\text { Riparian } \\
\text { Miles }\end{array}$ & $\begin{array}{r}2,016 \\
(46 \%)\end{array}$ & 271 & 1,140 & 60 & $\begin{array}{r}1,471 \\
(34 \%)\end{array}$ & $\begin{array}{r}698 \\
(16 \%)\end{array}$ & $\begin{array}{r}162 \\
(4 \%)\end{array}$ & 4,347 \\
\hline & $\begin{array}{l}\text { Wetland } \\
\text { Acres }\end{array}$ & $\begin{array}{r}4,879 \\
(63 \%)\end{array}$ & 10 & 589 & 105 & $\begin{array}{r}704 \\
(9 \%)\end{array}$ & 0 & $\begin{array}{r}2,130 \\
(27 \%)\end{array}$ & 7,713 \\
\hline \multirow[t]{2}{*}{ ES } & $\begin{array}{l}\text { Riparian } \\
\text { Miles }\end{array}$ & 0 & 0 & 0 & 0 & 0 & 0 & $\begin{array}{r}10 \\
(100 \%)\end{array}$ & 10 \\
\hline & $\begin{array}{l}\text { Wetland } \\
\text { Acres }\end{array}$ & 0 & 0 & 0 & 0 & 0 & 0 & $\begin{array}{r}4,300 \\
(100 \%) \\
\end{array}$ & 4,300 \\
\hline \multirow[t]{2}{*}{ ID } & $\begin{array}{l}\text { Riparian } \\
\text { Miles }\end{array}$ & $\begin{array}{r}1120 \\
(29 \%)\end{array}$ & 199 & 862 & 85 & $\begin{array}{r}1146 \\
(30 \%)\end{array}$ & $\begin{array}{r}397 \\
(10 \%)\end{array}$ & $\begin{array}{r}1,220 \\
(31 \%)\end{array}$ & 3,883 \\
\hline & $\begin{array}{l}\text { Wetland } \\
\text { Acres }\end{array}$ & $\begin{array}{r}1,071 \\
(8 \%)\end{array}$ & 117 & 1,107 & 100 & $\begin{array}{r}1,324 \\
(10 \%)\end{array}$ & $\begin{array}{r}248 \\
(2 \%) \\
\end{array}$ & $\begin{array}{r}10,317 \\
(80 \%)\end{array}$ & 12,960 \\
\hline \multirow[t]{2}{*}{ MT } & $\begin{array}{l}\text { Riparian } \\
\text { Miles }\end{array}$ & $\begin{array}{l}2,048 \\
(42 \%)\end{array}$ & 207 & 1,902 & 116 & $\begin{array}{r}2,225 \\
(46 \%)\end{array}$ & $\begin{array}{r}523 \\
(11 \%)\end{array}$ & $\begin{array}{r}57 \\
(1 \%)\end{array}$ & 4,853 \\
\hline & $\begin{array}{l}\text { Wetland } \\
\text { Acres }\end{array}$ & $\begin{array}{r}4,444 \\
(7 \%)\end{array}$ & 70 & 593 & 30 & $\begin{array}{r}693 \\
(1 \%)\end{array}$ & $\begin{array}{r}859 \\
(1 \%)\end{array}$ & $\begin{array}{r}56,518 \\
(91 \%)\end{array}$ & 62,514 \\
\hline
\end{tabular}

Attachment 1-4 
Table 2-2. CONDITION OF RIPARIAN-WETLAND AREAS, FY 1998 --continued

\begin{tabular}{|c|c|c|c|c|c|c|c|c|c|}
\hline \multirow[t]{2}{*}{ State } & \multirow{2}{*}{$\begin{array}{l}\text { Habitat } \\
\text { Types }\end{array}$} & \multirow{2}{*}{$\begin{array}{c}\text { Proper } \\
\text { Functioning } \\
\text { Condition }\end{array}$} & \multicolumn{4}{|c|}{ Functioning-At-Risk } & \multirow{2}{*}{$\begin{array}{c}\text { Non } \\
\text { Functional }\end{array}$} & \multirow[t]{2}{*}{ Unknown } & \multirow[t]{2}{*}{ Total } \\
\hline & & & $\begin{array}{c}\text { Trend } \\
\text { Up }\end{array}$ & $\begin{array}{c}\text { Trend } \\
\text { Not } \\
\text { Apparent }\end{array}$ & $\begin{array}{l}\text { Trend } \\
\text { Down }\end{array}$ & Total & & & \\
\hline \multirow[t]{2}{*}{ NV } & $\begin{array}{l}\text { Riparian } \\
\text { Miles }\end{array}$ & $\begin{array}{r}582 \\
(25 \%)\end{array}$ & 347 & 349 & 218 & $\begin{array}{r}914 \\
(40 \%)\end{array}$ & $\begin{array}{r}297 \\
(13 \%)\end{array}$ & $\begin{array}{r}501 \\
(22 \%)\end{array}$ & 2,244 \\
\hline & $\begin{array}{l}\text { Wetland } \\
\text { Acres }\end{array}$ & $\begin{array}{r}7,283 \\
(22 \%)\end{array}$ & 209 & 749 & 304 & $\begin{array}{r}1,262 \\
(4 \%)\end{array}$ & $\begin{array}{r}4,087 \\
(13 \%)\end{array}$ & $\begin{array}{r}20,014 \\
(61 \%)\end{array}$ & 32,646 \\
\hline \multirow[t]{2}{*}{$\mathrm{NM}$} & $\begin{array}{l}\text { Riparian } \\
\text { Miles }\end{array}$ & $\begin{array}{r}160 \\
(35 \%)\end{array}$ & 80 & 105 & 33 & $\begin{array}{r}218 \\
(48 \%)\end{array}$ & $\begin{array}{r}73 \\
(16 \%)\end{array}$ & $\begin{array}{r}5 \\
(1 \%)\end{array}$ & 456 \\
\hline & $\begin{array}{l}\text { Wetland } \\
\text { Acres }\end{array}$ & $\begin{array}{r}1,680 \\
(12 \%)\end{array}$ & 6 & 2 & 2 & $\begin{array}{r}10 \\
\text { (trace) }\end{array}$ & $\begin{array}{r}777 \\
(5 \%) \\
\end{array}$ & $\begin{array}{r}11,864 \\
(83 \%) \\
\end{array}$ & 14,331 \\
\hline \multirow[t]{2}{*}{ OR } & $\begin{array}{l}\text { Riparian } \\
\text { Miles }\end{array}$ & $\begin{array}{l}2,150 \\
(32 \%)\end{array}$ & 1,130 & 412 & 93 & $\begin{array}{r}1,635 \\
(24 \%)\end{array}$ & $\begin{array}{r}127 \\
(2 \%)\end{array}$ & $\begin{array}{r}2,802 \\
(42 \%)\end{array}$ & 6,714 \\
\hline & $\begin{array}{l}\text { Wetland } \\
\text { Acres }\end{array}$ & $\begin{array}{r}105,421 \\
(69 \%)\end{array}$ & 1,545 & 1,128 & 272 & $\begin{array}{r}2,945 \\
(2 \%)\end{array}$ & $\begin{array}{r}478 \\
\text { (trace) }\end{array}$ & $\begin{array}{r}44,867 \\
(29 \%)\end{array}$ & 153,711 \\
\hline \multirow[t]{2}{*}{ UT } & $\begin{array}{l}\text { Riparian } \\
\text { Miles }\end{array}$ & $\begin{array}{r}1,720 \\
(35 \%)\end{array}$ & 501 & 787 & 212 & $\begin{array}{r}1,500 \\
(30 \%)\end{array}$ & $\begin{array}{r}390 \\
(8 \%) \\
\end{array}$ & $\begin{array}{r}1,312 \\
(27 \%)\end{array}$ & 4,922 \\
\hline & $\begin{array}{l}\text { Wetland } \\
\text { Acres }\end{array}$ & $\begin{array}{r}5,184 \\
(36 \%)\end{array}$ & 3,134 & 336 & 71 & $\begin{array}{r}3,541 \\
(25 \%)\end{array}$ & $\begin{array}{r}470 \\
(3 \%)\end{array}$ & $\begin{array}{r}5,207 \\
(36 \%)\end{array}$ & 14,402 \\
\hline \multirow[t]{2}{*}{ WY } & $\begin{array}{l}\text { Riparian } \\
\text { Miles }\end{array}$ & $\begin{array}{r}1,279 \\
(27 \%)\end{array}$ & 792 & 802 & 562 & $\begin{array}{r}2,156 \\
(45 \%)\end{array}$ & $\begin{array}{r}624 \\
(13 \%)\end{array}$ & $\begin{array}{r}703 \\
(15 \%)\end{array}$ & 4,762 \\
\hline & $\begin{array}{l}\text { Wetland } \\
\text { Acres }\end{array}$ & $\begin{array}{r}6,590 \\
(32 \%) \\
\end{array}$ & 1,250 & 2,918 & 1,978 & $\begin{array}{r}6,146 \\
(30 \%) \\
\end{array}$ & $\begin{array}{r}225 \\
(1 \%) \\
\end{array}$ & $\begin{array}{r}7,500 \\
(37 \%) \\
\end{array}$ & 20,461 \\
\hline \multirow{2}{*}{$\begin{array}{c}\text { Total } \\
\text { Lower } \\
48\end{array}$} & $\begin{array}{l}\text { Riparian } \\
\text { Miles }\end{array}$ & $\begin{array}{r}13,230 \\
(36 \%)\end{array}$ & 4,067 & 7,280 & 1,553 & $\begin{array}{r}12,900 \\
(35 \%)\end{array}$ & $\begin{array}{r}3,251 \\
(9 \%)\end{array}$ & $\begin{array}{r}7,310 \\
(20 \%)\end{array}$ & 36,691 \\
\hline & $\begin{array}{l}\text { Wetland } \\
\text { Acres }\end{array}$ & $\begin{array}{r}147,923 \\
(41 \%) \\
\end{array}$ & 27,271 & 13,951 & 3,913 & $\begin{array}{r}45,135 \\
(13 \%) \\
\end{array}$ & $\begin{array}{r}7,557 \\
(2 \%) \\
\end{array}$ & $\begin{array}{r}166,819 \\
(44 \%) \\
\end{array}$ & 367,434 \\
\hline \multirow[t]{2}{*}{$\begin{array}{l}\text { Total } \\
\text { BLM }\end{array}$} & $\begin{array}{l}\text { Riparian } \\
\text { Miles }\end{array}$ & $\begin{array}{r}141,105 \\
(80 \%)\end{array}$ & 4,102 & 7,280 & 1,553 & $\begin{array}{r}12,935 \\
(7 \%)\end{array}$ & $\begin{array}{r}4,063 \\
(2 \%)\end{array}$ & $\begin{array}{r}18,949 \\
(11 \%)\end{array}$ & 177,052 \\
\hline & $\begin{array}{l}\text { Wetland } \\
\text { Acres }\end{array}$ & $\begin{array}{r}12,524,123 \\
(97 \%)\end{array}$ & 27,271 & 13,951 & 3,913 & $\begin{array}{r}45,135 \\
\text { (trace) }\end{array}$ & $\begin{array}{c}7,557 \\
\text { (trace) }\end{array}$ & $\begin{array}{r}355,619 \\
(3 \%)\end{array}$ & $12,932,434$ \\
\hline
\end{tabular}

Table 2-2. CONDITION OF RIPARIAN-WETLAND AREAS, FY 1998--concluded 


\section{FOOTNOTES}

/a/ The BLM's definition of riparian areas excludes stream reaches where water flows for only brief periods during storm runoff events (ephemeral streams). Original estimates of riparian extent were based on generalized United States Geological Survey stream network information. Intensive field assessments have provided additional data that has been used to exclude ephemeral stream reaches and refine estimates, thereby reducing the total number of riparian miles. The reduction in wetland area estimates is a result of advances in mapping technology used in Alaska. Greater accuracy in classifying and measuring resources is possible using remote sensing techniques, various sources of imagery, and Geographic Information System (GIS) computer technology.

/b/ Riparian and wetland areas are functioning properly when adequate vegetation, landform, or large woody debris is present to dissipate stream energy associated with high waterflows.

/c/ "Functioning-At-Risk" areas are functioning properly, but an existing soil, water, or vegetation attribute makes them susceptible to degradation. The trend is an assessment of apparent direction of change in conditions either towards or away from the site potential or site stability. Trend is determined by comparing the present condition with previous photos, trend studies, inventories, other documentation, or personal knowledge. The lack of historical information on the condition of a site may lead to a "trend not apparent" assessment.

/d/ "Nonfunctional" areas do not contain sufficient vegetation, landform, or large woody debris to dissipate stream energy associated with high flows.

/e/ "Unknown" areas have not been assessed by the BLM.

/f/ Riparian areas are green zones along flowing water features such as rivers, streams, and creeks (also referred to as lotic habitat areas), and are reported in miles.

/g/ Wetland areas are associated with standing water features such as bogs, marshes, wet meadows, and estuaries (also referred to as lentic habitat areas), and are reported in acres.

/h/ Alaska's wetland functioning-at-risk trend is unknown. 